\title{
What cosmology can tell us about neutrino physics in the next 10 years?
}

\author{
Sergio Pastor*i \\ Instituto de Física Corpuscular (CSIC-Universitat de València) \\ Ed. Institutos de Investigación, Apdo. 22085, E-46071 Valencia, Spain \\ E-mail: Sergio.Pastor@ific.uv.es
}

\begin{abstract}
Neutrinos can play an important role in the evolution of the Universe, modifying some of the cosmological observables. We describe how the precision of present cosmological data can be used to learn about neutrino properties, in particular their mass. Cosmology can provide information on the absolute scale of neutrino masses, complementary to the results of tritium beta decay and neutrinoless double beta decay experiments. We show how the analysis of current cosmological observations provides an upper bound on the sum of neutrino masses, with improved sensitivity from future cosmological measurements well into the sub-eV range.
\end{abstract}

10th International Workshop on Neutrino Factories, Super beams and Beta beams

June 30 - July 52008

Valencia, Spain

\footnotetext{
${ }^{*}$ Speaker.

${ }^{\dagger}$ Supported by the European Union under the ILIAS project (contract No. RII3-CT-2004-506222) and by the Spanish grant FPA2005-01269.
} 


\section{Introduction}

Neutrino cosmology is one of the best examples of the very close ties that have developed between nuclear physics, particle physics, astrophysics and cosmology. This contribution is focused on the information that the analysis of cosmological data can provide on neutrino masses. For a more detailed discussion of neutrinos in cosmology, see the reviews $[1,2,3]$.

\section{The cosmic neutrino background}

The existence of the cosmic neutrino background (CNB) is a generic feature of the standard hot big-bang model. Although not detected yet, the presence of the CNB is indirectly established through the accurate agreement between the calculated and observed primordial abundances of light elements, as well as from the analysis of the power spectrum of CMB anisotropies. Here we review its evolution and main properties.

At large temperatures frequent weak interactions kept cosmic neutrinos of any flavour $\left(v_{e, \mu, \tau}\right)$ in equilibrium until these processes became ineffective in the course of the expansion. While coupled to the rest of the primeval plasma (relativistic $e^{ \pm}$and photons), neutrinos had a FermiDirac spectrum with temperature $T, f_{\mathrm{eq}}(p, T)=[\exp (p / T)+1]^{-1}$. As the Universe cools, the weak interaction rate falls below the expansion rate and neutrinos decouple from the rest of the plasma, at a decoupling temperature $T_{\mathrm{dec}} \simeq 1 \mathrm{MeV}$. After decoupling the momentum spectrum is preserved, since both neutrino momenta and temperature redshift identically with the expansion. Since active neutrino masses are not much larger than $1 \mathrm{eV}$, they were ultra-relativistic at $T_{\text {dec }}$ and the spectrum does not depend on neutrino masses even after decoupling.

Shortly after the temperature drops below the electron mass, favouring the annihilations of $e^{ \pm}$that transfer their entropy into photons but not into the decoupled neutrinos. This leads to $T_{\gamma} / T_{v}=(11 / 4)^{1 / 3} \simeq 1.40102$, the ratio between the temperatures of relic photons and neutrinos. It turns out that a detailed calculation of neutrino decoupling leads to small non-thermal distortions in the neutrino spectra and a slightly smaller increase of the comoving photon temperature. The most recent analysis [4] includes the effect of flavour neutrino oscillations, and finds that these distortions lead to a contribution of relativistic relic neutrinos to the total energy density of $N_{\mathrm{eff}} \simeq 3.046$.

Any quantity related to relic neutrinos can be calculated after decoupling. For instance, the number density per flavour $n_{v}=(3 / 11) n_{\gamma}=\left(6 \zeta(3) / 11 \pi^{2}\right) T_{\gamma}^{3}$ is fixed by the temperature, leading to a present value of 339 neutrinos and antineutrinos per $\mathrm{cm}^{3}$. Instead, the energy density for massive neutrinos must instead be calculated numerically, with two well-defined analytical limits: $\rho_{v}=\left(7 \pi^{2} / 120\right)(4 / 11)^{4 / 3} T_{\gamma}^{4}\left(m_{v} \ll T_{v}\right)$ and $\rho_{v}=m_{v} n_{v}\left(m_{v} \gg T_{v}\right)$.

\section{Massive neutrinos in cosmology}

Nowadays there exist compelling evidences for non-zero neutrino masses from the experimental data on flavour neutrino oscillations [5]. Oscillation experiments measure two differences of squared neutrino masses, and the $3 \sigma$ ranges from [6] are $\left|\Delta m_{31}^{2}\right|=\left|m_{3}^{2}-m_{1}^{2}\right|=\left(2.40_{-0.33}^{+0.35}\right) \times$ $10^{-3} \mathrm{eV}^{2}$ and $\Delta m_{21}^{2}=m_{2}^{2}-m_{1}^{2}=\left(7.65_{-0.60}^{+0.69}\right) \times 10^{-5} \mathrm{eV}^{2}$. Unfortunately oscillation experiments are insensitive to the absolute scale of neutrino masses, and two schemes are still possible, known 
as normal $(\mathrm{NH})$ and inverted $(\mathrm{IH})$ hierarchies, characterized by the sign of $\Delta m_{31}^{2}$. For small values of the lightest neutrino mass $m_{0}$, i.e. $m_{1}\left(m_{3}\right)$ for $\mathrm{NH}(\mathrm{IH})$, the mass states follow a hierarchical scenario, while for masses much larger than the differences all neutrinos share in practice the same mass and then we say that they are degenerate. In general, the relation between the individual masses and their sum is found numerically.

Cosmology is at first order sensitive to the sum of neutrino masses but blind to mixing angles or possible $\mathrm{CP}$ violating phases, while laboratory experiments such as neutrinoless double beta decay and tritium beta decay are sensitive to combinations of masses and mixing parameters called $m_{\beta \beta}$ and $m_{\beta}$. Since the current limits from tritium beta decay are $m_{\beta} \lesssim 2.2 \mathrm{eV}$ (95\% CL) from the Troitsk and Mainz experiments, the sum of neutrino masses is restricted to the approximate range $0.06(0.1) \lesssim \sum_{i} m_{i} / \mathrm{eV} \lesssim 6$. The allowed regions in the parameter space defined by any pair of parameters $\left(\sum_{i} m_{i}, m_{\beta \beta}, m_{\beta}\right)$ can be found in $[7,8]$.

Do neutrino oscillations have an effect on any cosmological epoch? In the standard picture all flavour neutrinos were produced with the same energy spectrum, so no effect is expected from oscillations among these three states (up to small spectral distortions, see [4]). But there are two cases where neutrino oscillations could have cosmological consequences: flavour oscillations with non-zero relic neutrino asymmetries and active-sterile neutrino oscillations (see e.g. Sec. 5 in [9]).

A priori, massive neutrinos are excellent candidates for being the Dark Matter, the dominant non-baryonic component of the matter density in the Universe. We know that they exist and their energy density in units of the critical value is $\Omega_{v}=\rho_{v} / \rho_{\mathrm{c}}^{0}=M_{v} /\left(93.14 h^{2} \mathrm{eV}\right)$, where $h \equiv H_{0} /\left(100 \mathrm{~km} \mathrm{~s}^{-1} \mathrm{Mpc}^{-1}\right)$ is the present value of the Hubble parameter. The neutrino density fraction is usually defined with respect to the total matter density, $f_{v} \equiv \Omega_{v} / \Omega_{\mathrm{m}}$. The total mass $M_{v} \equiv \sum_{i} m_{i}$ includes all masses of the neutrino states which are non-relativistic today, at least two because both $\left(\left|\Delta m_{31}^{2}\right|\right)^{1 / 2} \simeq 0.05 \mathrm{eV}$ and $\left(\Delta m_{21}^{2}\right)^{1 / 2} \simeq 0.009 \mathrm{eV}$ are larger than $T_{v} \simeq 1.7 \times 10^{-4} \mathrm{eV}$. The range of present values of $\Omega_{v}$ compatible with oscillation data and the approximate bounds from beta decay experiments is $0.0013(0.0022) \lesssim \Omega_{v} \lesssim 0.13$ (for $h \approx 0.7$ ).

In general, a cosmological upper bound on $\Omega_{v}$ has been used since the 1970 s to constrain the possible values of neutrino masses. If we demand that neutrinos should not be too heavy to overclose the Universe $\left(\Omega_{v}<1\right)$, we obtain an upper bound $M_{v} \lesssim 45 \mathrm{eV}$. Moreover, since from present analyses of cosmological data we know that is $\Omega_{\mathrm{m}} \simeq 0.3$, the neutrino masses should obey the stronger bound $M_{v} \lesssim 15 \mathrm{eV}$, which is roughly only a factor 2 worse than that from tritium decay.

Particles with a large velocity dispersion such as that of neutrinos are called hot dark matter (HDM), and they affect the evolution of cosmological perturbations in a particular way: the density contrasts are erased on wavelengths smaller than a mass-dependent free-streaming scale. If HDM dominates, this suppression contradicts various observations and this is why the attention turned to cold dark matter (CDM) candidates. Still in the mid-1990s it appeared that adding a small contribution of HDM fitted better the observational data than a pure CDM model. However, within the presently favoured $\Lambda \mathrm{CDM}$ model dominated at late times by a cosmological constant (or some form of dark energy) there is no need for a significant HDM contribution. Instead, we use the available cosmological data to find how large neutrino masses can be. 


\section{Effects of neutrino masses on cosmology}

Here we describe very briefly the effects caused by neutrino masses on the main cosmological observables. For a more detailed discussion, see [3].

In general, neutrino masses of the order of $1 \mathrm{eV}$ (about $f_{v} \leq 0.1$ ) were still relativistic at the time of recombination between electrons and nucleons, i.e. after photon decoupling. Thus they only affect the shape of the power spectrum of CMB temperature anisotropies through the modified background evolution, related to the fact that these neutrinos account today as matter but to radiation at that time [3]. This changes some characteristic times and scales in the cosmological evolution, and affects mainly the amplitude of the first acoustic peak as well as the location of all peaks. Thus, it is possible to constrain neutrino masses using CMB data alone [3,10], down to the level at which this background effect is masked by instrumental noise or cosmic variance, or by parameter degeneracies in extended models (such as varying the radiation content of the Universe).

The current Large Scale Structure (LSS) of the Universe is probed by the matter power spectrum, observed with various techniques. It is defined as the two-point correlation function of nonrelativistic matter fluctuations in Fourier space $P(k, z)=\left\langle\left|\delta_{\mathrm{n}}(k, z)\right|^{2}\right\rangle$, where the matter perturbation $\delta_{\mathrm{m}}=\sum_{i} \bar{\rho}_{i} \delta_{i} / \sum_{i} \bar{\rho}_{i}$, includes all components (CDM, baryons, non-relativistic neutrinos, ...) and represents indifferently the energy or mass power spectrum of non-relativistic matter. The shape of $P(k)$ is affected by the free-streaming caused by small neutrino masses and thus it is the key observable for constraining $m_{v}$. The physical effect of free-streaming is to damp small-scale neutrino density fluctuations: neutrinos cannot be confined into (or kept outside of) regions smaller than the free-streaming length. Instead, on scales much larger, the neutrino velocity effectively vanishes and after the non-relativistic transition the neutrino perturbations behave like CDM: modes with $k<k_{\mathrm{nr}}=0.018 \Omega_{\mathrm{m}}^{1 / 2}(\mathrm{~m} / \mathrm{eV})^{1 / 2} h \mathrm{Mpc}^{-1}$ [3], are never affected by free-streaming and evolve like in a pure $\Lambda \mathrm{CDM}$ model. There exist additional effects of massive neutrinos related to the background evolution and a gravitational back-reaction effect that also damp the metric perturbations on those scales (see Sec. 4.5 of [3]). The combined effect of neutrino masses is an attenuation of small-scale perturbations for $k>k_{\mathrm{nr}}$, as shown in Fig. 13 of [3]. For small values of $f_{v}$ this effect is approximated in the large $k$ limit by the well-known linear expression $P(k)^{f_{v}} / P(k)^{f_{v}=0} \simeq 1-8 f_{v}$ [11].

Is it possible to mimic the effect of massive neutrinos on $P(k)$ with some combination of other cosmological parameters? Ideally, if we could measure $P(k)$ on a large interval of $k$ values, the effect of neutrino masses would be non-degenerate, because of its very characteristic steplike shape. The problem is that usually $P(k)$ can only be accurately measured in the intermediate region where the mass effect is neither null nor maximal. In this region, neutrino masses affect the slope of the matter power spectrum in a way which can be easily confused with the effect of other cosmological parameters. Therefore, we need to combine LSS data with other cosmological data, in particular the CMB anisotropy spectrum, which could lift most of the degeneracies.

\section{Current bounds on neutrino masses}

Here we review how the available cosmological data is used to get information on the absolute scale of neutrino masses, complementary to laboratory experiments. Note that the bounds in the next subsections are all based on the Bayesian inference method, and the upper bounds on the sum 
of neutrino masses are given at 95\% C.L. after marginalization over all free cosmological parameters. We refer the reader to section 5.1 of [3] for a detailed discussion. Here it is assumed that the total neutrino mass is the only additional parameter with respect to a flat $\Lambda \mathrm{CDM}$ cosmological model characterized by 6 parameters, unless specified otherwise.

\subsection{CMB anisotropies}

The experimental situation of the measurement of the CMB anisotropies is dominated by the fifth-year release of WMAP data (WMAP5, see [12]), which improved the already precise TT and TE angular power spectra of the previous releases (WMAP1 ad WMAP3), and adds a detection of the E-polarization self-correlation spectrum (EE). On similar or smaller angular scales than WMAP, we have results from experiments that are either ground-based (ACBAR, VSA, CBI, DASI, ...) or balloon-borne (ARCHEOPS, BOOMERANG, MAXIMA, ....).

We saw in the previous section that the signature on the CMB spectrum of a neutrino mass smaller than $0.5 \mathrm{eV}$ is small but does not vanish due to a background effect, proportional to $\Omega$, which changes some characteristic times and scales in the evolution of the Universe, and affects mainly the amplitude of the first acoustic peak as well as the location of all the peaks. Therefore, it is possible to constrain neutrino masses using CMB experiments only. In this framework, many analyses support the conclusion that a sensible bound on neutrino masses exists using CMB data only, of order of $2-3 \mathrm{eV}$ for the total mass. This is an important result, since it does not depend on the uncertainties from LSS data discussed next.

\subsection{Galaxy redshift surveys}

We have seen that free-streaming of massive neutrinos produces a direct effect on the formation of cosmological structures. Actually, it is well known that the presence of neutrino masses leads to an attenuation of the linear matter power spectrum on small scales. In a seminal paper [11] it was shown that an efficient way to probe neutrino masses of order $\mathrm{eV}$ was to use data from large redshift surveys, which measure the distance to a large number of galaxies, giving us a three-dimensional picture of the universe. At present, we have data from two large projects: the 2 degree Field (2dF) galaxy redshift survey and the Sloan Digital Sky Survey (SDSS).

One of the main goals of galaxy redshift surveys is to reconstruct the power spectrum of matter fluctuations on very large scales, whose cosmological evolution is described entirely by linear perturbation theory. However, the linear power spectrum must be reconstructed from individual galaxies which underwent a strongly non-linear evolution. A simple analytic model of structure formation suggests that on large scales, the galaxy-galaxy correlation function should be, not equal, but proportional to the linear matter density power spectrum, up to a constant factor that is called the light-to-mass bias $(b)$. This parameter can be obtained from independent methods, which tend to confirm that the linear biasing assumption is correct, at least in first approximation.

A conservative way to use the measurements of galaxy-galaxy correlations in an analysis of cosmological data is to take the bias as a free parameter, i.e. to consider only the shape of the matter power spectrum at the corresponding scales and not its amplitude (denoted as galaxy clustering data). An upper limit on $M_{v}$ between 0.8 and $1.7 \mathrm{eV}$ is found from the analysis of galaxy clustering data (SDSS and/or 2dF, leaving the bias as a free parameter) added to CMB data. These values 
improve those found with CMB data only. The bounds on neutrino masses are more stringent when the amplitude of the matter power spectrum is fixed with a measurement of the bias, instead of leaving it as a free parameter. The upper limits on $M_{v}$ are reduced to values of order $0.5-0.9$ $\mathrm{eV}$ although some analyses also add Lyman- $\alpha$ data (see next subsection).

Finally, a galaxy redshift survey performed in a large volume can also be sensitive to the imprint created by the baryon acoustic oscillations (BAO) at large scales on the power spectrum of non-relativistic matter. Since baryons are only a subdominant component of the non-relativistic matter, the BAO feature is manifested as a small single peak in the galaxy correlation function in real space that was recently detected from the analysis of the SDSS luminous red galaxy (LRG) sample. The observed position of this baryon oscillation peak provides a way to measure the angular diameter distance out to the typical LRG redshift of $z=0.35$, which in turn can be used to constrain the parameters of the underlying cosmological model.

\subsection{Lyman- $\alpha$ forest}

The matter power spectrum on small scales can also be inferred from data on the so-called Lyman- $\alpha$ forest. This corresponds to the Lyman- $\alpha$ absorption of photons traveling from distant quasars $(z \sim 2-3)$ by the neutral hydrogen in the intergalactic medium. As an effect of the Universe expansion, photons are continuously red-shifted along the line of sight, and can be absorbed when they reach a wavelength of $1216 \AA$ in the rest-frame of the intervening medium. Therefore, the quasar spectrum contains a series of absorption lines, whose amplitude as a function of wavelength traces back the density and temperature fluctuations of neutral hydrogen along the line of sight. It is then possible to infer the matter density fluctuations in the linear or quasi-linear regime.

In order to use the Lyman- $\alpha$ forest data, one needs to recover the matter power spectrum from the spectrum of the transmitted flux, a task that requires the use of hydro-dynamical simulations for the corresponding cosmological model. This is a difficult procedure, and given the various systematics involved in the analysis the robustness of Lyman- $\alpha$ forest data is still a subject of intense discussion between experts. In any case, the recovered matter power spectrum is again sensitive to the suppression of growth of mass fluctuations caused by massive neutrinos, and in many cosmological analyses the Lyman- $\alpha$ data is added to CMB and other LSS data. For a free bias, one finds that Lyman- $\alpha$ data help to reduce the upper bounds on the total neutrino mass to the level $M_{v}<0.5-0.7 \mathrm{eV}$. But those analyses that include Lyman- $\alpha$ data and a measurement of the bias do not always lead to a lower limit, ranging from 0.4 to $0.7 \mathrm{eV}$.

\subsection{Summary and discussion of current bounds}

The upper bounds on $M_{v}$ from the previous subsections are representative of an important fact: a single cosmological bound on neutrino masses does not exist. Depending on the included set of data, the approximate ranges for the upper bounds are: $2-3 \mathrm{eV}$ for $\mathrm{CMB}$ only, $0.9-1.7 \mathrm{eV}$ for $\mathrm{CMB}$ and $2 \mathrm{dF} / \mathrm{SDSS}$-gal or $0.2-0.9 \mathrm{eV}$ with the inclusion of a measurement of the bias and/or Lyman- $\alpha$ forest data and/or the SDSS measurement of the baryon oscillation peak. For a recent discussion on the bounds on neutrino masses from different combinations of cosmological data, we refer the reader to [8]. In any case, current cosmological data probe the region of neutrino masses where the 3 neutrino states are degenerate, with a mass $M_{v} / 3$. It is interesting to compare these bounds with those coming from tritium beta decay and neutrinoless double beta decay [7]. 

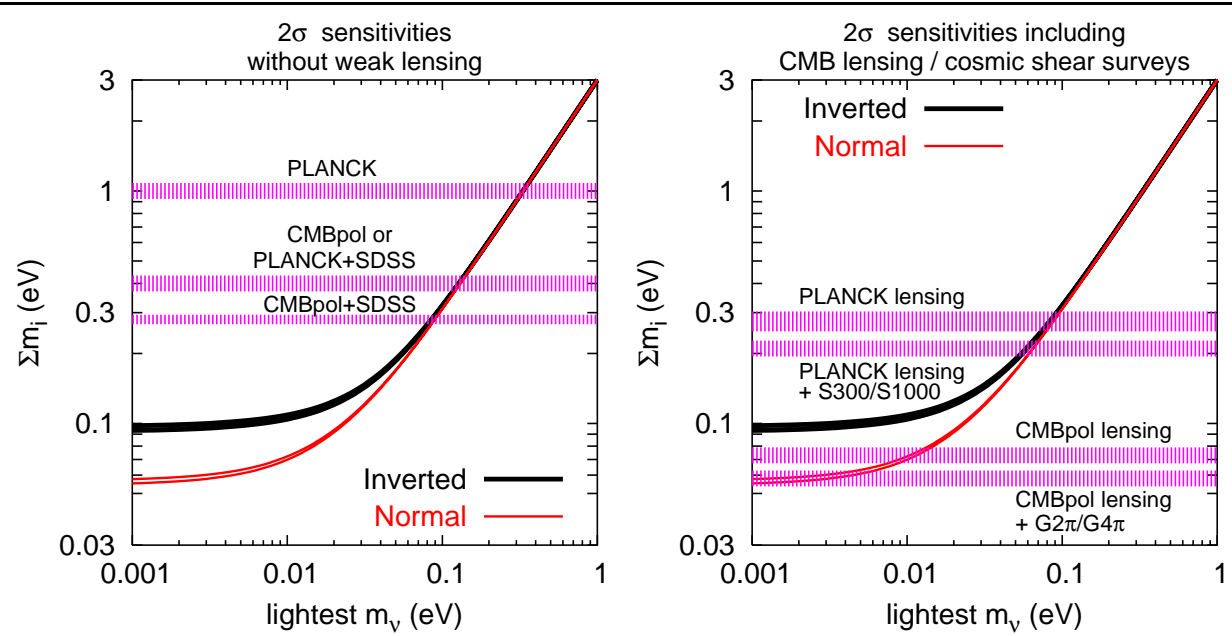

Figure 1: Forecast $2 \sigma$ sensitivities to the total neutrino mass from future cosmological experiments compared to the values in agreement with neutrino oscillation data (assuming a future determination at the 5\% level). Left: sensitivities expected for future CMB experiments (without lensing extraction), alone and combined with the completed SDSS galaxy redshift survey. Right: sensitivities expected for future CMB experiments including lensing information, alone and combined with future cosmic shear surveys. Here CMBpol refers to a hypothetical CMB experiment roughly corresponding to the INFLATION PROBE mission.

\section{Future sensitivities on neutrino masses from cosmology}

In the near future we will have more precise data on cosmological observables from various experimental techniques and experiments. If the characteristics of these future experiments are known with some precision, it is possible to assume a "fiducial model", i.e. a cosmological model that would yield the best fit to future data, and to estimate the error bar on a particular parameter that will be obtained after marginalizing the hypothetical likelihood distribution over all the other free parameters. Technically, the simplest way to forecast this error is to compute a Fisher matrix, a technique has been widely used in the literature, for many different models and hypothetical datasets, now complemented by Monte Carlo methods. Here we will focus on the results for $\sigma\left(M_{v}\right)$, the forecast $68 \% \mathrm{CL}$ error on the total neutrino mass, assuming various combinations of future observations: $\mathrm{CMB}$ anisotropies measured with ground-based experiments or satellites such as PLANCK, galaxy redshift surveys, galaxy cluster surveys, ... In particular, it has been recently emphasized the potentiality for measuring small neutrino masses of weak lensing experiments, which will look for the lensing effect caused by the large scale structure of the neighboring universe, either on the CMB signal [13] or on the apparent shape of galaxies (measured by cosmic shear surveys, see e.g. [14]). We refer the reader to section 6 of [3] for further details.

We give a graphical summary of the forecast sensitivities to neutrino masses of different cosmological data in fig. 1, compared to the allowed values of neutrino masses in the two possible 3-neutrino schemes. One can see from this figure that there are very good prospects for testing neutrino masses in the degenerate and quasi-degenerate mass regions above $0.2 \mathrm{eV}$ or so. A detection at a significant level of the minimal value of the total neutrino mass in the inverted hierarchy scheme will demand the combination of future data from CMB lensing and cosmic shear surveys, whose more ambitious projects will provide a $2 \sigma$ sensitivity to the minimal value in the case of nor- 
mal hierarchy (of order $0.05 \mathrm{eV}$ ). The combination of $\mathrm{CMB}$ observations with future galaxy cluster surveys [15] or the measurement of the redshifted $21 \mathrm{~cm}$ signal from the epoch of reionization using low-frequency radio observations [16], should yield similar or even better sensitivities.

\section{Conclusions}

Neutrinos, despite the weakness of their interactions and their small masses, can play an important role in Cosmology. In addition, cosmological data can be used to constrain neutrino properties, providing information on these elusive particles that complements the efforts of laboratory experiments. In particular, the data on cosmological observables have been used to bound the effective number of neutrinos (including a potential extra contribution from other relativistic particles). But probably the most important contribution of Cosmology to our knowledge of neutrino properties is the information it can provide on the absolute scale of neutrino masses. We have seen that the analysis of cosmological data can lead to either a bound or a measurement of the sum of neutrino masses, an important result complementary to terrestrial experiments such as tritium beta decay and neutrinoless double beta decay experiments. In the next future, thanks to the data from new cosmological experiments we could even hope to test the minimal values of neutrino masses guaranteed by the present evidences for flavour neutrino oscillations. Therefore, we expect that neutrino cosmology will remain an active research field in the next years.

\section{References}

[1] A.D. Dolgov, Phys. Rep. 370 (2002) 333 [arXiv:hep-ph/0202122].

[2] S. Hannestad, Ann. Rev. Nucl. Part. Sci. 56 (2006) 137 (2006) [arXiv:hep-ph/0602058].

[3] J. Lesgourgues and S. Pastor, Phys. Rep. 429 (2006) 307 [arXiv:astro-ph/0603494].

[4] G. Mangano et al, Nucl. Phys. B 729 (2005) 221 [arXiv:hep-ph/0506164].

[5] C. González-García, in proceedings of 10th International Workshop on Neutrino Factories, Super beams and Beta beams (NuFact08), June 30 - July 5 2008, Valencia, Spain PoS (NUFACT08) 002.

[6] T. Schwetz, M. Tórtola and J.W.F. Valle, arXiv:0808.2016

[7] G.L. Fogli et al, Phys. Rev. D 75 (2007) 053001 [arXiv:hep-ph/0608060].

[8] G.L. Fogli et al, Phys. Rev. D 78 (2008) 033010 [arXiv:0805.2517].

[9] S. Pastor, lectures at 61st SUSSP (Ed. Taylor \& Francis Academic Pub., 2007), in press.

[10] K. Ichikawa, M. Fukugita and M. Kawasaki, Phys. Rev. D 71 (2005) 043001 [arXiv:astro-ph/0409768].

[11] W. Hu, D.J. Eisenstein and M. Tegmark, Phys. Rev. Lett. 80 (1998) 5255 [astro-ph/9712057].

[12] E. Komatsu et al [WMAP Collaboration], arXiv:0803.0547

[13] J. Lesgourgues, L. Perotto, S. Pastor and M. Piat, Phys. Rev. D 73 (2006) 045021 [astro-ph/0511735].

[14] S. Hannestad, H. Tu and Y.Y.Y. Wong, JCAP 0606 (2006) 025 [arXiv:astro-ph/0603019].

[15] S. Wang et al, Phys. Rev. Lett. 95 (2005) 011302 [astro-ph/0505390].

[16] J.R. Pritchard and E. Pierpaoli, Phys. Rev. D 78 (2008) 065009 [arXiv:0805.1920]. 